Pacific Journal of Mathematics

F - 3 RINGS WITH ZERO SINGULAR IDEAL

Robert Ray Colby and Edgar Andrews Butter 


\title{
QF-3 RINGS WITH ZERO SINGULAR IDEAL
}

\author{
R. R. Colby and E. A. Rutter, JR.
}

Let $R$ be a ring with identity. $R$ is left $Q F-3$ if $R$ has a minimal faithful (left) module, i.e., a faithful (left) module, which is (isomorphic to) a summand of every faithful (left) module. We show that left $Q F-3$ rings are characterized by the existence of a faithful projective-injective left ideal with an essential socle which is a finite sum of simple modules. The main result is a structure theorem for left and right $Q F-3$ rings with zero left singular ideal. This theorem gives several descriptions of this class of rings. Among these is that the above rings are exactly the orders (containing units) with essential left and right socles in semi-simple two-sided (complete) quotient rings.

Let $R$ be a ring with identity. $R$ is left $Q F-3$ if $R$ has a minimal faithful (left) module, i.e., a faithful (left) module which is (isomorphic to) a summand of every faithful (left) module. Finite dimensional algebras with this property were introduced by R. M. Thrall as a generalization of quasi-Frobenius algebras, and other authors have considered Artinian and semi-primary $Q F-3$ rings. For a semi-primary ring, being left $Q F-3$ is equivalent to the existence of a faithful projective-injective left ideal. The first theorem shows that to characterize left $Q F-3$ rings in general one must assume that some faithful projective-injective left ideal has an essential socle which is a finite sum of simple modules. The rest of this paper concerns rings with zero singular ideal which are either $Q F-3$ or have faithful projectiveinjective one-sided ideals. The main result is a structure theorem for left and right $Q F-3$ rings with zero left singular ideal. This theorem gives several descriptions of this class of rings. Among these is that the above rings are exactly the orders (containing units) with essential left and right socles in semi-simple ${ }^{1}$ two-sided (complete) quotient rings. This theorem extends and unifies result of M. Harada [5, 6], J. P. Jans [8], and H. Mochizuki [12].

REsults. A submodule $N$ of a module $M$ is essential in $M$ if every nonzero submodule of $M$ meets $N$ nontrivially. The singular submodule $Z(M)=\{x \in M \mid I x=0$ for some essential left ideal $I$ of $R\}$. $Z\left({ }_{R} R\right)$ is an ideal of $R$ called the left singular ideal of $R$. An $R$ module $M$ is called uniform if every nonzero submodule of $M$ is essential in $M$. If $M$ and $N$ are $R$-modules with $M$ uniform and

1 Throughout this paper, semi-simple means semi-simple Artinian. 
$Z(N)=0$, then every nonzero homomorphism of $M$ into $N$ is a monomorphism (see Goldie [4]). In particular, if $M$ is injective and $N$ is indecomposable, every such homomorphism is an isomorphism.

The structure of a minimal faithful $R$-module is given by the following theorem. We denote the injective envelope of an $R$-module $M$ by $E(M)$ (see [1] or [11]).

THEOREM 1. The following are equivalent.

(1) $R$ is left $Q F-3$.

(2) There exist (nonisomorphic) simple (left) $R$-modules $S_{1}, \cdots$, $S_{n}$ such that $E\left(\oplus_{i=1}^{n} S_{i}\right)$ is a faithful, projective module.

(3) There exist (nonisomorphic) minimal left ideals $M_{1}, \cdots, M_{k}$ of $R$ such that $E\left(\bigoplus_{i=1}^{k} M_{i}\right)$ is a faithful left ideal of $R$.

Proof. It is clear that (3) implies (2). Thus it suffices to show (1) implies (3) and (2) implies (1).

Assume (1). Let $M$ be the minimal faithful module. Since ${ }_{R} R$ is faithful, $M$ is isomorphic to a summand of ${ }_{R} R$ and so is projective and cyclic. Let $\left\{S_{\alpha}: \alpha \in A\right\}$ be a complete set of representatives for the distinct isomorphic classes of simple left $R$-modules. Then $\bigoplus_{\alpha} E\left(S_{\alpha}\right)$ is faithful (see [14]) and so $M$ is isomorphic to a summand of $\oplus_{\alpha} E\left(S_{\alpha}\right)$. Since $M$ is cyclic, its image is contained in a finite number of the $E\left(S_{\alpha}\right)$. Thus $M$ is injective and has an essential socle which is the direct sum of a finite number of simple modules.

Assume (2). Then since $E\left(\bigoplus_{i=1}^{n} S_{i}\right) \cong \bigoplus_{i=1}^{n} E\left(S_{i}\right)$ each $E\left(S_{i}\right)$ is an indecomposable projective-injective module and so is isomorphic to a left ideal $L_{i}$ of $R$ (see [2]) which must contain a unique minimal left ideal $M_{i}$. One now shows that $\bigoplus_{i=1}^{n} L_{i}$ is a minimal faithful module as in $[8]$.

Lemma 2. Suppose that $R$ e is a faithful projective-injective left ideal of $R$ where $e^{2}=e \in R$. If either (a) eRe is semi-simple Artinian or (b) $Z(R)=0$ and $R$ contains no infinite set of orthogonal idempotents, then

(1) ReR is the right socle of $R$ and contains only a finite number of isomorphism classes of simple right $R$-modules.

(2) ReR is an essential right ideal of $R$.

(3) the right singular ideal of $R$ is zero.

Proof. Since $R e$ is injective and $e R e$ is the endomorphism ring of $R e$, in either case we have $R e=R e_{1} \oplus \cdots \oplus R e_{n}$ where the $e_{i}$ 's are orthogonal idempotents and each $R e_{i}$ is indecomposable and injective. Hence each $e_{i} R e_{i}$ is a local ring. In case (a), the radical of $e_{i} R e_{i}$ is 
$e_{i}(\operatorname{rad} e R e) e_{i}=0$ so $e_{i} R e_{i}$ is a division ring. This also follows if (b) holds since $R e_{i}$ is uniform and injective and $Z\left(R e_{i}\right)=0$. Let $J$ denote the radical of $R$. If (a) holds then $e_{i} J=0$ for each $i$ since $R e$ is faithful. If (b) holds and $e_{i} x \neq 0$ with $x \in J$, then $r e_{i} \rightarrow r e_{i} x$ defines a monomorphism of $R e_{i}$ into $J$ whose image is a summand of $R$, a contradiction. Hence $e_{i} R$ contains no nilpotent right ideals, so $e_{i} R$ is simple as in ([7], Prop. 1, p. 65). Hence $R e_{i} R$ is contained in the right socle of $R$ and so $R e R$ is also. Now, if $H$ is a right ideal with $R e R \cap H=0$, then $H R e \subseteq R e R \cap H$ so since $R e$ is faithful $H=0$. Thus $R e R$ is an essential submodule of $R_{R}$ and so equals the right socle of $R$. Since $R e R$ is a faithful left $R$-module the right singular ideal of $R$ is zero.

If $R$ is a subring of a ring $Q$ such that ${ }_{R} R$ is essential in ${ }_{R} Q$, then $Q$ is called a ring of left quotients of $R$.

Proposition 3. Suppose $R$ contains faithful projective-injective left and right ideals $R e$ and $f R$, respectively, where $e$ and $f$ are idempotents. Then $\operatorname{Hom}_{e R e}(R e, f R e)=f R$ and $\operatorname{Hom}_{f R f}(f R, f R e)=R e$. Furthermore, $Q=\operatorname{Hom}_{\text {ene }}(R e, R e)$ is a two-sided ring of quotients of $R$.

Proof. Let $Q=\operatorname{Hom}_{e R e}(R e, R e)$. Since the map $\lambda$ of $R$ into $Q$ given by $\lambda(r)(s e)=r s e$ for $r, s \in R$ is a unital ring monomorphism whose restriction to $R e$ is a $Q$-monomorphism, we may regard $R$ as a subring of $Q$ such that $R e=Q e$ is a faithful left $Q$-module. Since $R e$ is faithful $f R_{R}$ is an essential submodule of $f Q_{R}$. Thus, since $f R_{R}$ is injective, $f Q=f R$. Since $\operatorname{Hom}_{e R e}(R e, f R e)=f Q$ the first assertion holds. Now ${ }_{Q} R e$ is faithful and since if $q \in Q$ and $f R q=0$, then $q R e=0, f R_{Q}$ is faithful. Thus, if $0 \neq q \in Q, q R \cap R \supseteqq q R e \neq 0$ and $R q \cap R \supseteqq f R q \neq 0$ so $Q$ is a two-sided quotient ring of $R$.

Lemma 4. Suppose that $R$ contains faithful projective-injective left and right ideals $R e$ and $f R$ where $e$ and $f$ are idempotents, and that $f R f$ is semi-simple Artinian. Then $R e_{e R e}$ is not an infinite direct sum of nonzero submodules.

Proof. Supppose $R e=\bigoplus_{j} I_{j}$ where each $I_{j}$ is a nonzero $e R e$ module. Then $\operatorname{Hom}_{e R e}(R e, f R e)=\Pi_{j} \mathrm{Hom}_{e R e}\left(I_{j}, f R e\right)=f R$. Suppose the direct sum is infinite so there exists $f r \in f R$ such that $f r \notin \bigoplus_{j} \operatorname{Hom}_{e R e}$ $\left(I_{j}, f R e\right)$. Since ${ }_{f R f} f R e$ is faithful and injective and $f R f$ is semi-simple, there exists $s e \in R e=\operatorname{Hom}_{f R f}(f R, f R e)$ such that frse $\neq 0$ and $\left(\oplus_{j} \operatorname{Hom}_{f R f}\left(I_{j}, f R e\right)\right) s e=0$, a contradiction.

An $R$-module $M$ is finite dimensional if $M$ contains no infinite direct sum of nonzero submodules (see [3[). The complete ring of left 
quotients of $R$ is $\operatorname{Hom}_{\Gamma}\left(E\left({ }_{R} R\right)_{\Gamma}, E\left({ }_{R} R\right)_{\Gamma}\right)$ where $\Gamma=\operatorname{Hom}_{R}\left(E\left({ }_{R} R\right), E\left({ }_{R} R\right)\right)$ (see Lambek [10] or [11]). The complete ring of left quotients of $R$ is semi-simple if and only if ${ }_{R} R$ is finite dimensional and $R$ has zero left singular ideal (Johnson [9]).

If $M$ is an $R$-module we say that $N$ is a minimal essential submodule of $M$ if $N$ is essential in $M$ and no proper submodule of $N$ is essential in $M$.

We are now ready for the main theorem.

THEOREM 5. The following are equivalent.

(1) $R$ has zero left singular ideal and is left and right $Q F-3$.

(2) There exist idempotents $e, f \in R$ such that $R e$ and $f R$ are faithful projective-injective left and right ideals and eRe is semisimple Artinian.

(3) $R$ has zero left singular ideal, contains no infinite set of orthogonal idempotents and has a faithful projective-injective left ideal and a faithful projective-injective right ideal.

(4) $R$ is a subring of a semi-simple Artinian ring $Q$ and $R$ contains a left ideal $I$ and a right ideal $J$ such that $I$ and $J$ are, respectively, faithful left and faithful right ideals of $Q$.

(5) $R$ has a two-sided semi-simple Artinian complete ring of quotients and both the left socle and the right socle of $R$ are essential.

(6) $R$ has a two-sided semi-simple Artinian complete ring of quotients and ${ }_{R} R$ and $R_{R}$ each contain a minimal essential submodule.

Proof. Conditions (5) and (6) are equivalent since the socle of any module is the intersection of its essential submodules (see Utumi [16]). We complete the proof by showing first that (1) and (2) are equivalent. and then that $(1) \rightarrow(5) \rightarrow(4) \rightarrow(3) \rightarrow(1)$.

Assume condition (1). If $R e$ is a minimal faithful left ideal with $e^{2}=e$ then Theorem 1 together with $Z(R)=0$ imply that the endomorphism ring of $R e$ is semi-simple so (2) holds.

Assume condition (2). By Lemma 2 the right singular ideal of $R$ is zero and the right socle $R e R$ of $R$ is essential in $R_{R}$. Since there are only a finite number of isomorphism classes of simple right ideals of $R, f R$ contains a minimal faithful module by Theorem 1 . Hence $R$ is right $Q F-3$ with zero singular ideal and if we take $f R$ to be a minimal faithful, $f R f$ is semi-simple. Thus by an argument symmetric to the above, $R$ is left $Q F-3$ and has zero left singular ideal. Hence (1) holds.

Assume condition (1). Let $R e, e^{2}=e$, and $f R, f^{2}=f$ be minimal faithful left and right ideals of $R$, respectively. Then both the left and the right socles of $R$ are essential in $R$ by Lemma 2. By Proposition $3, Q=\operatorname{Hom}_{e R e}(R e, R e)$ is a two-sided ring of quotients of $R$. 
That $Q$ is semi-simple is plain from Lemma 4 since both $e R e$ and $f R f$ are semi-simple.

Next assume condition (5) holds. Let $Q$ denote the two-sided semi-simple quotient ring of $R$ and let $E$ denote the left socle of $R$. Then $E Q=E$ since $E$ is the left socle of ${ }_{R} Q$. Furthermore, since the left singular ideal of $R$ is zero and $Q_{R}$ is essential over $R_{R}, E$ is a faithful right $Q$-module and so we let $J=E$. Similarly, we can let $I$ be the right socle of $R$. Thus (4) holds.

Assume condition (4). Then $Q_{R}$ is a right ring of quotients of $R$ since for any $0 \neq q \in Q, 0 \neq q I \subseteq R$ and hence since $Q$ is semi-simple, it is the complete ring of right quotients of $R$. Similarly, $Q$ is the complete ring of left quotients of $R$. Next note that ${ }_{R} I$ is injective. For, if $f: L \rightarrow I$ where $L$ is a left ideal of $R$, define $\bar{f}: Q L \rightarrow I=Q I$ by

$$
\bar{f}\left(\sum q_{i} x_{i}\right)=\sum q_{i} f\left(x_{i}\right), \quad q_{i} \in Q, \quad x_{i} \in L .
$$

$\bar{f}$ is well defined since if $E$ is the left socle of $R, y \in E$ and $\sum q_{i} x_{i}=0$ then $y \sum q_{i} f\left(x_{i}\right)=f\left(\sum\left(y q_{i} x_{i}\right)\right)=0$ so $Z\left({ }_{R} Q\right)=0$ implies $\sum q_{i} f\left(x_{i}\right)=0$. Thus $\bar{f}$ is a $Q$-homomorphism and injectivity of ${ }_{R} I$ follows from the injectivity of ${ }_{Q} I$. Similarly, $J$ is a faithful projective-injective right ideal of $R$ so (3) holds.

Finally, condition (1) follows from condition (3) by Theorem 1 and Lemma 2.

REMARK 6. If $R$ satisfies the hypotheses of Theorem 5 and $R e, e^{2}=e$, and $f R, f^{2}=f$, are faithful projective-injective left and right ideals of $R$, respectively, then $f R e$ character modules define a duality between finitely generated right $e R e$-modules and finitely generated $f R f$-modules in the sense of Morita [13]. The semi-simplicity of $e R e$ and $f R f$ with the faithfulness of ${ }_{f R f} f R e$ and $f R e_{e R e}$ implies that ${ }_{f R f} f R e$ and $f R e_{e R e}$ are injective cogenerators. The mapping of $f R$ onto $f R e$ given by right multiplication by $e$ induces an injection of $\operatorname{Hom}_{f R f}(f R e, f R e)$ into $\operatorname{Hom}_{f R f}(f R, f R e)=R e$ which has image $e R e$.

It is not difficult to show that if $R$ has zero left singular ideal, then $R$ is left $Q F-3$ and finite dimensional if and only if $R$ is left and right $Q F-3$. Thus Theorem 5 extends results of $\mathrm{H}$. Mochizuki [12] for hereditary $Q F-3$ algebras and M. Harada [5, 6] for semiprimary $Q F-3$ and $P P$ rings. This theorem also generalizes results of J. P. Jans [8] for primitive rings with faithful projective-injective minimal one-sided ideals.

A ring is (meet) irreducible if the intersection of an two nonzero ideals is nonzero. If $R$ is left $Q F-3$ and the socle of $R$ is homogeneous, then the socle of a minimal faithful left ideal is simple and hence 
is contained in every nonzero two-sided ideal. The following corollary to Theorem 5 is easily proved.

COROLlaRY 7. Let $R$ be a ring which satisfies the conditions of Theorem 5 and let $Q$ denote its complete ring of quotients. The following are equivalent.

(1) $R$ is the direct sum of ideals $R_{1}, \cdots, R_{n}$ and $Q$ is the direct sum of ideals $Q_{1}, \cdots, Q_{n}$ where each $Q_{i}$ is a two-sided simple Artinian complete quotient ring of $R_{i}$.

(2) $R$ is the direct sum of ideals $R_{1}, \cdots, R_{n}$ where each $R_{i}$ is an irreducible ring.

(3) $R$ has a decomposition $R=R e_{1} \oplus \cdots \oplus R e_{m}$ into indecomposable left ideals $R e_{i}$ such that each $R e_{i}$ has homogeneous socle.

It is not difficult to show that if (3) above holds, any such decomposition has the stated property. In (2) above the intersection of the left and right socles of $R_{i}$ is the minimal ideal of $R_{i}$.

\section{REFERENCES}

1. B. Eckmann, and A. Schopf, Uber injective moduln, Arch. Math. 4 (1953), 75-78.

2. C. Faith, and E. A. Walker, Direct sum representations of injective modules, J. A. 5 (1967), 203-221.

3. A. W. Goldie, Semi-prime rings with maximum condition, Proc. London Math. Soc.(3) 10 (1960), 201-220.

4. Torsion-free modules and rings, J. A. 1 (1964), 268-287.

5. M. Harada, On semi-primary PP-rings, Osaka J. Math. 2 (1965), 153-161.

6. - $-Q F-3$ and semi-primary PP-rings I and II, Osaka J. Math. 2 (1965), 357368 and 3 (1966), 21-27.

7. N. Jacobsen, Structure of rings, Amer. Math. Soc. Colloq. Pub. Vol. 36,'Providence, R. I., 1964.

8. J. P. Jans, Projective-injective modules, Pacific J. Math. 9 (1958), 1103-1108.

9. R. E. Johnson, Quotient rings of rings with zero singular ideal, Pacific J. Math. 11 (1961), 1385-1392.

10. J. Lambek, On Utumi's ring of quotients, Canad. J. Math. 15 (1963), 363-370.

11. Lectures on rings and modules, Blaisdell Pub. Co., Wâltham, Mass., 1966.

12. H. Y. Mochizuki, On the double commutator algebra of QF-3 algebras, Nagoya Math. J. 25 (1965), 221-230.

13. K. Morita, Duality for modules and its applications to the theory of rings with minimum condition, Tokyo Kyoiku Daigaku, Sec A. 6 (1958), 83-142.

14. A. Rosenberg, and D. Zelinsky, Finiteness of the injective hull, Math. Z. 70 (1959), $372-380$.

15. R. M. Thrall, Some generalizations of quasi-Frobenius algebras, Trans. Amer. Math. Soc. 64 (1948), 173-183.

16. Y. Utumi, Y. Self injective rings, J. A. 6 (1967), 54-67.

Received April 15, 1968.

The UNIVERSity of Kansas

LAWRENCE, KANSAS 


\section{PACIFIC JOURNAL OF MATHEMATICS}

\section{EDITORS}

H. ROYDEN

Stanford University

Stanford, California

\section{R. $R$ PHELPS}

University of Washington

Seattle, Washington 98105

\section{J. DugunduI}

Department of Mathematics

University of Southern California

Los Angeles, California 90007

RICHARD ARENS

University of California

Los Angeles, California 90024

\section{ASSOCIATE EDITORS}

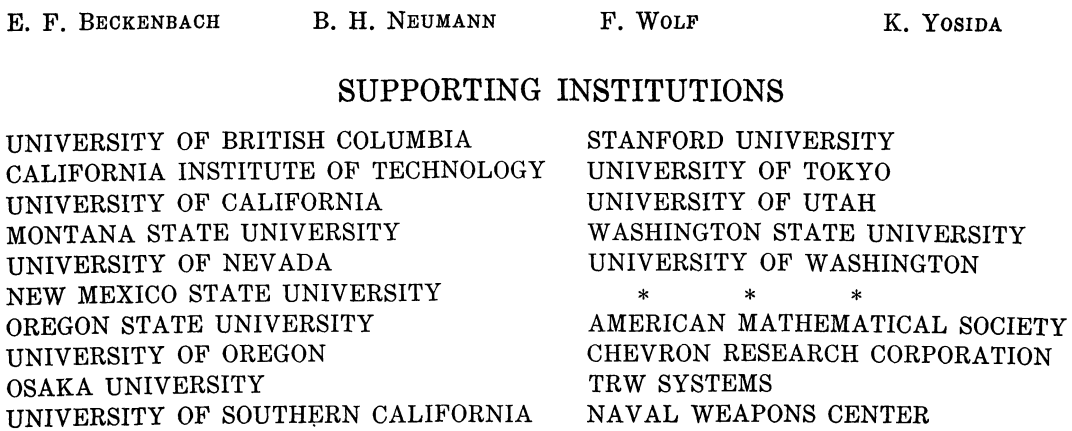

The Supporting Institutions listed above contribute to the cost of publication of this Journal, but they are not owners or publishers and have no responsibility for its content or policies.

Mathematical papers intended for publication in the Pacific Journal of Mathematics should be in typed form or offset-reproduced, double spaced with large margins. Underline Greek letters in red, German in green, and script in blue. The first paragraph or two must be capable of being used separately as a synopsis of the entire paper. It should not contain references to the bibliography. Manuscripts, in duplicate if possible, may be sent to any one of the four editors. Please classify according to the scheme of Math. Rev. 36, 1539-1546. All other communications to the editors should be addressed to the managing editor, Richard Arens, University of California, Los Angeles, California, 90024.

50 reprints are provided free for each article; additional copies may be obtained at cost in multiples of 50 .

The Pacific Journal of Mathematics is published monthly. Effective with Volume 16 the price per volume (3 numbers) is $\$ 8.00$; single issues, $\$ 3.00$. Special price for current issues to individual faculty members of supporting institutions and to individual members of the American Mathematical Society: $\$ 4.00$ per volume; single issues $\$ 1.50$. Back numbers are available.

Subscriptions, orders for back numbers, and changes of address should be sent to Pacific Journal of Mathematics, 103 Highland Boulevard, Berkeley, California, 94708.

PUBLISHED BY PACIFIC JOURNAL OF MATHEMATICS, A NON-PROFIT CORPORATION

Printed at Kokusai Bunken Insatsusha (International Academic Printing Co., Ltd.), 7-17, Fujimi 2-chome, Chiyoda-ku, Tokyo, Japan. 


\section{Pacific Journal of Mathematics}

Vol. 28, No. $2 \quad$ April, 1969

Richard Arens and Donald George Babbitt, The geometry of relativistic

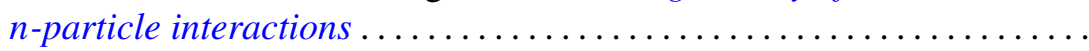

Kirby Alan Baker, Hypotopological spaces and their embeddings in lattices

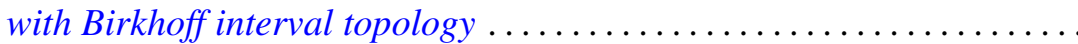

J. Lennart (John) Berggren, Finite groups in which every element is conjugate to its inverse ........................... 289

Beverly L. Brechner, Homeomorphism groups of dendrons . . . . . . . . . . . 295

Robert Ray Colby and Edgar Andrews Rutter, QF - 3 rings with zero singular ideal ................................. 303

Stephen Daniel Comer, Classes without the amalgamation property....... 309

Stephen D. Fisher, Bounded approximation by rational functions ......... 319

Robert Gaines, Continuous dependence for two-point boundary value problems..................................... 327

Bernard Russel Gelbaum, Banach algebra bundles ............... 337

Moses Glasner and Richard Emanuel Katz, Function-theoretic degeneracy criteria for Riemannian manifolds ...................... 351

Fletcher Gross, Fixed-point-free operator groups of order $8 \ldots \ldots \ldots \ldots 357$

Sav Roman Harasymiv, On approximation by dilations of distributions . . . . 363

Cheong Seng Hoo, Nilpotency class of a map and Stasheff's criterion ... . . 375

Richard Emanuel Katz, A note on extremal length and modutus.......... 381

H. L. Krall and I. M. Sheffer, Difference equations for some orthogonal polynomials ................................

Yu-Lee Lee, On the construction of lower radical properties ........... 393

Robert Phillips, Liouville's theorem........................... 397

Yum-Tong Siu, Analytic sheaf cohomology groups of dimension $n$ of

n-dimensional noncompact complex manifolds ..... . .

Michael Samuel Skaff, Vector valued Orlicz spaces. II...

James DeWitt Stein, Homomorphisms of $B^{*}$-algebras .... . .

Mark Lawrence Teply, Torsionfree injective modules .... . . .

Richard R. Tucker, The $\delta^{2}$-process and related topics. II .

David William Walkup and Roger Jean-Baptiste Robert Wets, Lifting

projections of convex polyhedra...

Thomas Paul Whaley, Large sublattices of a lattice. 